\title{
ASYMPTOMATIC BACTERIURIA IN HIV/AIDS PATIENTS: OCCURRENCE AND RISK ASSOCIATED WITH LOW CD4 COUNTS.
}

\author{
Rashmi. K.S, Ravikumar. K.L, Jagadeesh, Nimitha, Bhagyashree. H.N.
}

1. Assistant Professor, Department of Microbiology, KIMSH\&RC, Bangalore

2. Professor and HOD, Department of Microbiology, KIMSH\&RC, Bangalore

3. Professor, Department of Microbiology, KIMSH\&RC, Bangalore

4. Junior Research Fellow, Department of Microbiology, KIMSH\&RC, Bangalore

5. Tutor, Department of Microbiology, KIMSH\&RC, Bangalore

\section{CORRESPONDING AUTHOR:}

Rashmi. K. S.

Assistant Professor, Department of Microbiology,

Kempegowda Institute of Medical Sciences and Research Center,

KR Road, VV Puram, Bangalore-560004

E-mail: rashminaga05@gmail.com

ABSTRACT: BACKGROUND: People living with HIV/AIDS with progressive immune dysfunction have an augmented risk of Urinary tract infection, both symptomatic and asymptomatic, in comparison to non-infected individuals. AIMS: This prospective study was planned to know the frequency of asymptomatic bacteruria (ASB) in HIV sero-positives and its correlation to their immune status. Conventional culture method was compared with screening test using SD Urocolor TM 10 test strips for the presence of infection. Antibiogram of isolated uropathogens were analysed to understand the trends of drug resistance. SETTINGS AND DESIGN: Prospective Cohort Study. MATERIALS AND METHODS: Clean catch midstream urine samples collected from 270 HIV seropositive patients and 100 seronegative controls were screened for ASB by Urostrip urinalysis, Gram stain and culture. Antibiogram of significant growth isolates was done by Kirby-Bauer disc diffusion method. Their immune status was evaluated by CD4 + cell counting. STATISTICAL ANALYSIS USED: Sensitivity, Specificity, Odds ratio, Fisher extract test. RESULTS: ASB was detected in $5.6 \%(15 / 270)$ of HIV sero-positives and $1 \%(1 / 100)$ of seronegatives controls.13/15 HIV seropositive ASB cases had CD4 cell counts < 350 cells/ $\mu \mathrm{L}$. The gram stain and urostrips had sensitivity of $93.33 \% \& 80 \%$ and a specificity of $95.68 \%$ \& $60.39 \%$ respectively. CONCLUSIONS: Occurrence of ASB was significantly high among the HIV sero-positives with CD4 cell counts < 350 cells $/ \mu \mathrm{L}$. Conventional culture and gram staining continue to be the proficient methods for detecting ASB. Cotrimaxazole prophylaxis hardly influenced the presence of ASB in HIV sero-positives.

KEY-WORDS: Asymptomatic bacteriuria, HIV, CD4 counts, Antibiogram.

INTRODUCTION: India is estimated to bear the largest load of HIV infected on account of the size of its population with an advanced stage of epidemic in some of its states 1 . Untreated UTI accounting for 7-60\% of opportunistic infections could be a source for ascending urinary tract infection and septicaemia in immunocompromised hosts 2, 3, 4. As limited data is available from India on the frequency of ASB/UTIs in HIV seropositives, the present study aimed to assess its occurrence by culture and screening test. Antibiogram of significant urinary isolates was performed to understand the prevalence of drug resistance. 
MATERIALS AND METHODS: STUDY DESIGN \&STUDY SITE: This prospective observational hospital based study was conducted over a period of twelve weeks (September 2012 to November2012). Adult outpatient HIV positive patients accessing care in the antiretroviral (ART) clinic of KIMS Hospital and Research Centre, Bangalore are included.HIV patients with symptoms of urinary tract infection were excluded from study. Urine samples were obtained from 270 consecutive patients and 100 healthy individuals. Baseline demographic data, CD4 counts and information about cotrimaxazole prophylaxis of the patients were also collected. Written consent from the study participants and ethical approval from the institutional ethics committee was obtained.

URINE COLLECTION: Clean catch, midstream urine samples were collected aseptically after explanation by the nurse. Specimens were processed within 2 hours of collection. The samples were divided into two equal parts, the first part was processed for culture on UTI chrome agar and the second part was utilised for Grams stain and urostrip analysis. After overnight incubation at $37^{\circ} \mathrm{C}$ the plates were read and the growths were identified by standard methods. A bacterial count of $1 \times 10^{5} / \mathrm{ml}$ was considered significant for ASB while counts less than $1 \times 10^{5} / \mathrm{ml}$ were considered as no significant bacterial growth. A bacterial count of $1 \times 10^{4} / \mathrm{ml}$ was considered significant for gram positive organisms. Antimicrobial susceptibility tests were performed using Kirby-Bauer disc diffusion method 5,2 .

Urinalysis by chemical testing of urine was performed using SD Urocolor TM 10 test strips (urostrips) for Bilirubin, Urobilinogen, Nitrites, Specific gravity, protein, glucose, PH, erythrocyturia, leukocyturia and ketones according to manufacturer's instructions. Test strips were read manually at 60 seconds. The colour produced by the reaction was compared with the colour of the respective area on the reading scale. The result of this testing is regarded as semiquantitative ${ }^{6}$.

STATISTICAL ANALYSIS: Data was analysed using percentages, fisher exact test and Odds ratio. Differences were considered significant at $\mathrm{P} \leq 0.05^{7}$.

RESULTS: 370 samples were collected from 270 HIV seropositive and 100 HIV seronegative individuals. Growth was seen in 39/270(14.44\%) HIV positives of which ASB was present in 15 samples (5.6\%) The rest were insignificant or contaminant growth. In the control group significant growth was seen in 1/100 (1\%) samples. ASB was predominantly present in HIV seropositives with $\mathrm{p}$ value of 0.0358 . All the significant growth (15) is from sexually active individuals with age less than 50 years. Significant bacterial growth was more in females $(9 / 15)$ compared to males $(6 / 15)$ in this group.

Gram stain was positive in $8.51 \%$ (23/270) of HIV positives and 4\% (4/100) of HIV seronegatives. In HIV seropositives $14 / 23$ had significant growth and the rest were insignificant bacteriuria, contamination and no growth. Pus cells were found in 11 cases of which 6 were associated with ASB. Considering presence of nitrites, pyuria and proteinuria Urostrips were positive in $41.85 \%$ $(113 / 270)$ of HIV positives and 9\% (9/100) of HIV seronegatives (Table 1). 


\section{ORIGINAL ARTICAL}

Table-1

\begin{tabular}{|c|c|c|c|c|c|c|c|}
\hline \multirow{2}{*}{\multicolumn{2}{|c|}{ Parameters }} & \multicolumn{3}{|c|}{$\mathrm{HIV}+$} & \multicolumn{3}{|c|}{ HIV- } \\
\hline & & ASB+ve & ASB-ve & Total & ASB+ve & ASB-ve & Total \\
\hline \multirow[t]{2}{*}{ Gram stain } & Organism seen & 14 & 09 & 23 & 1 & 3 & 4 \\
\hline & Organism not seen & 1 & 246 & 247 & 0 & 96 & 96 \\
\hline \multirow[t]{2}{*}{ Pyuria } & Present & 4 & 61 & 65 & 1 & 2 & 3 \\
\hline & Absent & 11 & 194 & 205 & 0 & 97 & 97 \\
\hline \multirow[t]{2}{*}{ Nitrites } & Present & 6 & 81 & 87 & 0 & 0 & 0 \\
\hline & Absent & 9 & 174 & 185 & 1 & 99 & 100 \\
\hline \multirow[t]{2}{*}{ Proteinuria } & Present & 3 & 22 & 25 & 0 & 7 & 7 \\
\hline & Absent & 12 & 233 & 245 & 1 & 92 & 93 \\
\hline \multirow[t]{2}{*}{ Haematuria } & Present & 6 & 84 & 90 & 0 & 0 & 0 \\
\hline & Absent & 9 & 171 & 180 & 1 & 99 & 100 \\
\hline
\end{tabular}

Correlation of Gram staining and urinalysis (using SD urostrips) with ASB

The sensitivity and specificity of Gram's stain was $93.33 \%$ and $95.68 \%$ and that of urostrip was $80 \%$ and $60.39 \%$ in comparison to culture.

Staphylococcus aureus (6/15) was the predominant isolate followed by Enterococcus (4/15), Klebsiella (3/15) and E.coli (2/15).The organism isolated from HIV negative female patient was Staphylococcus aureus. 13/15 isolates were multidrug resistant (i.e. resistant to $\geq 3$ classes of antibiotics). Both gram negative and gram positive isolates were resistant to Ciprofloxacin (11/15), Cefepime (8/15), Amoxyclav (9/15), Cotrimaxazole (9/15) and Gentamicin (4/15). All gram negative bacilli were resistant to Ceftazidime and Amoxyclav and sensitive to Pipericillin/Tazobactum. All the gram positive cocci were sensitive to Tetracycline and Vancomycin. (Figure 1 and Figure2)

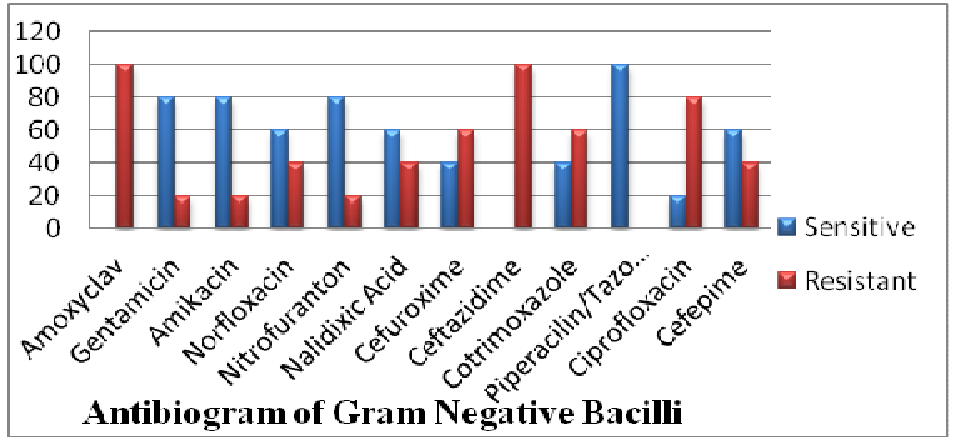

Figure1: Antibiotic profile of Gram Negative Bacilli

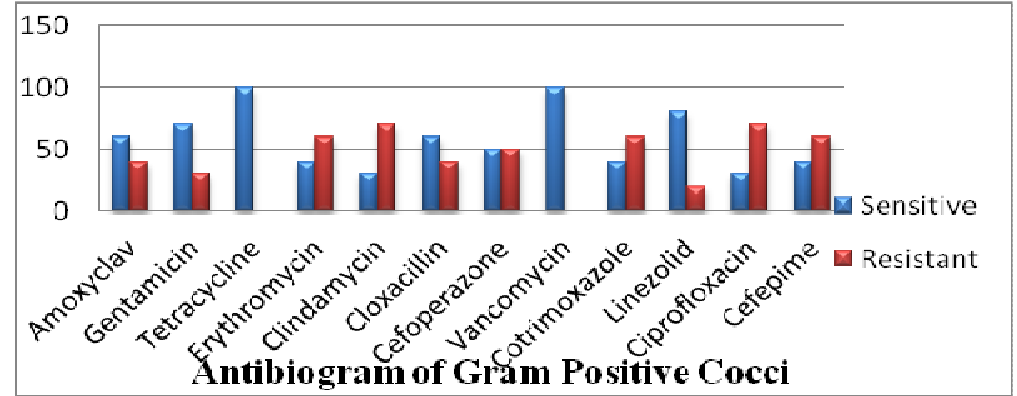

Figure 2: Antibiotic profile of Gram Positive Cocci 
Out of 270 patients 66 were on Cotrimaoxazole prophylaxis. 4/66(6\%) patients had significant growth of whom 3 were resistant to Cotrimaoxazole. Among 204 patients who were not on Cotrimaoxazole prophylaxis, 11 (5.4\%) showed ASB of whom 6 were resistant to Cotrimaoxazole. $60 \%(9 / 15)$ of the organisms isolated were resistant to Cotrimaoxazole. The cotrimaxalole resistant isolates were also resistant to ciprofloxacin (5/9) and amoxyclav (4/9). 8 /9 isolates were sensitive to gentamicin. All cotrimaxalole resistant gram positives were sensitive vancomycin, tetracycline and linezolid.

Among HIV seropositives, 137 patients had CD4 counts $<350 / \mu \mathrm{L}$ and $133 \mathrm{had}>350 / \mu \mathrm{L}$ $13 / 15$ patients with ASB had CD4 count $<350$ cells/ $\mu \mathrm{L}$. The risk of developing bacteriuria was 6.8 times greater in patients with CD4 counts $<350 / \mu \mathrm{L}(86 \%)$ with $\mathrm{P}$ value of 0.0123 .The mean CD4 count of the subjects who had ASB was $264 / \mu \mathrm{L}$ (Table 2)

Table2

\begin{tabular}{|c|c|c|}
\hline CD4 counts & No examined & Total No with ASB \\
\hline $0-100$ & 15 & 0 \\
\hline $100-350$ & 122 & 13 \\
\hline$>350$ & 133 & 2 \\
\hline Total & 270 & 15 \\
\hline
\end{tabular}

Correlation of ASB in HIV seropositives with their CD4 counts

DISCUSSION: Opportunistic infections are the cause of high morbidity and mortality in HIV/ AIDS patients. Prompt and accurate diagnosis and management of OIs will not only prolong the life of an HIV infected individual but also improve the quality of life. So an early and accurate diagnosis of the aetiological agent is important 1.

HIV disease is associated with a variety of renal syndromes. In patients with low CD4 counts bladder areflexia and hyporeflexia is a common neurologic complication, which leads to urinary stasis, and ultimately infection. Individuals with AIDS seem to be at an augmented risk of UTI, both symptomatic and asymptomatic, in comparison with asymptomatic HIV-infected and non-HIV infected patients 8 , 9 . Diverse studies across the globe have reported the incidence of asymptomatic bacteriuria in HIV patients as $3.1 \%$-to the maximum of $26 \%$ compared to an average prevalence of $0.04 \%$ in healthy population $3,9,10,11$. The present study reports asymptomatic bacteriuria (ASB) in $5.6 \%$ of HIV positive and $1 \%$ of HIV negative individuals with a p value of 0.0358 which parallels with the findings of OM Ibadin 9. It was observed ASB was more in females (9/15) compared to males $(6 / 15)$ in sexually active group with age $<50$ years. The mechanisms associated with HIV infection that may be responsible for the observed increase in the frequency of UTI remain to be established 12, 13. It can be explained on the basis of immunosuppression causing infections in various body systems including kidneys ${ }^{9}$. It is also recognised that local antibody production can afford some protection against bacterial invasion of the urinary tract in immunocompetent patients. Although polyclonal activation of B lymphocytes and hypergammaglobulinaemia are frequently found in patients with AIDS, antibody response to $\mathrm{T}$ cell dependent and independent antigens is commonly inadequate. This impairment in the humoral immune response to bacterial antigens may facilitate the adhesion of pathogenic bacteria to the uroepithelium of patients with AIDS 12,13. 
Urine dipstick testing, as opposed to urinary microscopy, is considered to be a reasonable alternative to urinalysis for diagnosis of acute uncomplicated cystitis ${ }^{14}$. In the present study, urostrips was positive in $41.85 \%(113 / 270)$ of HIV positives and 9\%(9/100) of HIV seronegatives and Gram's stain was positive in $9.6 \%(26 / 270)$ HIV Positives and 3\%(3/100) HIV negatives. Taking culture as the gold standard, the sensitivity and specificity of Urostrips was $80 \%$ and $60.69 \%$. The sensitivity and specificity of microscopy by Gram's stain examination was $93.33 \%$ and $95.45 \%$ respectively. Goossens et al. have opined that asymptomatic bacteriuria couldn't be accurately predicted either by microscopic examination or by the nitrite test using dipsticks ${ }^{3}$. RD McNair et al have reported 53\% false-negative rate in an obstetric population with dipstick screening of nitrite and leukocyte esterase 15. Quantitative analysis of the causative organism along with its drug resistance pattern and prognostic significance can be obtained with the urine culture despite being more time consuming and less sensitive if patients are on antibiotics or diuretics ${ }^{5}$. When screening for bacteriuria is appropriate, the quantitative urine culture remains the optimal screening test ${ }^{15}$.

Pyuria as an indication of inflammatory process and occurring significantly more among patients with UTI is at tandem with most reports on UTI. It may serve as screening tool in situations where culture is not immediately feasible. In immunocompetent individuals, pyuria is present in $87 \%$ of UTIs. In HIV patients it may also represent side effects of some of the anti retroviral drugs ${ }^{9}$. In this study the presence of pyuria was determined by measuring leucocyte esterase levels using urostrips. Pyuria was present in 65(24.07 \%) of HIV positive and $3(3 \%)$ of HIV negative individuals which parallels with the results of $4,13.26 .6 \%$ ( $4 / 15$ ) HIV positive patients with ASB had pyuria. Fabian et al have reported similar findings (29.1\%) whereas MM Buzayan has found no correlation between the degree of pyuria and a significant urine culture 4, 3. Pyuria, determined either by urinalysis or leukocyte esterase dipstick, is not specific for infection, and subjects with asymptomatic bacteriuria do not always have pyuria. Bacteriuria with gram-positive organisms is associated with lower levels of pyuria ${ }^{15}$. No published studies to date have investigated the causes of (sterile) leukocyturia in HIV infection. In HIV infected patients an additional potential cause of sterile leukocyturia may be interstitial nephritis, which can be directly due to HIV infection or caused by drugs used in its management-cotrimoxazole, atazanavir, tenofovir, indinavir, efavirenz, through a hypersensitivity reaction. In advanced disease, opportunistic genitourinary pathogens (parvovirus B19, herpesvirus, cytomegalovirus, polyomavirus, Candida spp, Aspergillus spp.) and genitourinary tuberculosis may be implicated. Though many causes of sterile leukocyturia exist, whether these are different in the HIV-infected population is unknown. Sterile leukocyturia may act as a clinical indicator for screening easily treatable conditions in this subgroup of patients 4

Mild to moderate proteinuria occurs in 38 to $82 \%$ of HIV-seropositive patients while nephrotic-like proteinuria is seen in approximately $10 \%{ }^{8}$. With urostrips, proteinuria was present in $9.25 \%$ (25/270) of HIV positive and 2\% (2/100) of HIV negative individuals. $12 \%(3 / 25)$ of these patients with proteinuria had ASB. Several studies have reported the prevalence of proteinuria in HIV infected patients in the range of 8 to $28 \% 4,9,16,17$. Preliminary studies suggest that discontinuation of Zidovudine in treatment of HIV associated nephropathy may cause an irreversible, rapid loss of renal function. In addition, numerous medications (Trimethoprim-sulfamethoxazole, Foscarnet, Cidofovir, and Acyclovir) taken by HIV-positive patients are nephrotoxic and are responsible for Acute Tubular Necrosis and Acute renal failure ${ }^{8}$. 
Hematuria is often detected on urinalysis in patients with HIV disease who are asymptomatic $^{8}$. Haematuria was found in $33 \%$ of HIV positives in the current study. Microscopic haematuria of $33.1 \%$ and $25 \%$ has been observed in the studies of Staiman VR et al and Fabian et al 4 , 8. ASB was present in $6.7 \%(6 / 90)$ of HIV positives with haematuria. A positive urine culture prevalence of $7.1 \%$ was found in the study of Fabian et al. Isolated microscopic hematuria has been described in HIV infection due to uncontrolled HIV replication in uroepithelial tissue ${ }^{4}$. Since hematuria was rarely found to be clinically significant in various studies, asymptomatic HIV-positive patients with microhematuria do not require urologic evaluation unless previous urologic history or abnormal renal function is present ${ }^{8}$.

Presence of nitrites is a widely used parameter for screening UTI. Nitrite test results showed high false positivity in HIV positive individuals. (31\% of whom only $7 \%$ had ASB). Nitrite was absent in the control group. The specific contribution of the nitrite test for the diagnosis of UTI was very low in findings of MM Buzayan ${ }^{3}$.Various studies have shown that the nitrite dipstick is subject to falsenegatives, because 4 to 6 hours is required for bacteria to convert nitrate to nitrite in bladder urine, and some infecting organisms are nitrite negative ${ }^{15}$.

The analysis of the reports has shown that patients with HIV had ASB more frequently than the controls. Besides the difference in the frequency, we also observed difference in the etiology. The various organisms isolated in the current study include Staphylococcus aureus (40\%), Enterococcus (27\%), Klebsiella (20\%) and E.coli (13\%). This observation correlates to the results of Inyang Etoh PC et al where Staphylococcus was the most common uropathogen ${ }^{18}$. These are in contrast to the observations of various studies who have reported E.coli, Klebsiella, Pseudomonas and Enterococcus as the most common urinary isolates $3,4,9,11,13,8,19$. The changing pattern of aetiology should be taken into consideration before initiating treatment for UTI in AIDS patients.

Resistance to several antimicrobial agents was prevalent among the isolates recovered from the study participants. Ampicillin and Trimethoprim-Sulfamethoxazole (Cotrimoxazole) resistance among urinary tract isolates has been reported with increasing frequencies. The Infectious Disease Society of America (IDSA) guidelines suggest that in community with Cotrimoxazole resistance rates of $\geq 10$ to $20 \%$ among UTI pathogens, alternative antimicrobial agents should be considered as firstline treatment for acute uncomplicated bacterial cystitis in women Given that the majority of therapy for UTIs is empiric and that uropathogens are demonstrating increasing antimicrobial resistance, it is recommended that antibiotic resistance profiling of uropathogens be carried out prior to commencement of therapy ${ }^{21}$.

This is the first report of antibiogram of urinary isolates of HIV infected persons from Karnataka. In the current study, Co-trimoxazole prophylaxis did not have significant effect in prevention of ASB. ASB was found in $6 \%$ of patients on Co-trimoxazole compared to $5 \%$ in those who were not on Co-trimoxazole prophylaxis. In addition resistance to Co-trimoxazole was higher $75 \%$ compared to54\%) in the group who were on prophylaxis. These findings concur with published results, confirming that cotrimoxazole prophylaxis may not protect against UTI, most likely because of rapid emergence of uropathogen resistance ${ }^{4}$. Prospective bigger studies are needed to clarify whether co-trimoxazole confers some benefit in protecting HIV seropositive individuals against UTI 13.

Journal of Evolution of Medical and Dental Sciences/ Volume 2/ Issue 19/ May 13, 2013

Page 3363 
Multidrug resistance was seen in 13/15 isolates. Both gram negative and gram positive isolates were resistant to Ciprofloxacin (73\%), Gentamicin (27\%), Amoxyclav (60\%), Co-trimoxazole $(60 \%)$ and Cefepime (53\%). In addition, gram negative bacilli were resistant to Cefuroxime (60\%), Ceftazidime (100\%), Nalidixic acid (40\%), Norfloxacin (40\%), Nitrofurantoin (20\%) and Amikacin (20\%). All were sensitive to Pipericillin/Tazobactum. Among the gram positives, $70 \%$ were resistant to clindamycin, $60 \%$ to erythromycin and $50 \%$ to Cefoperazone, $40 \%$ to Cloxacillin, and $20 \%$ to Linezolid. All were sensitive to Tetracycline and Vancomycin. The antibiotic profile is similar to the studies of Deokar S.D et al, Iweriebor B.C and Ojoo J et al 2, 20, 21. Prophylactic treatment of several opportunistic bacterial and parasitic infections using cotrimoxazole and penicillins has been ascribed to their increase resistance ${ }^{9}, 11$. The antibiotics susceptibility profiles of the isolated bacteria in the present study may be used to guide physicians in the selection of antibiotics for treating HIV patients. These results clearly demonstrate that a great care must be observed by physicians who are dealing with HIV infection when treatment of a UTI with suspected bacteraemia in AIDS patients is initiated ${ }^{3}$.

UTIs appears to be multi-factorial in subjects with HIV infection as the WHO stage of the disease, the CD4 level, and other complications of the urinary tract are significantly associated with UTI among subjects with HIV infection ${ }^{20}$. In the only controlled longitudinal study reported, it was found that the risk for bacteriuria was increased in HIV infected patients with CD4 + lymphocyte counts below 200 cells/mm3 when compared with two other groups of HIV infected patients with higher CD4 + lymphocyte counts ${ }^{12}$. In the present study it was observed that the patients with immunosuppression had a very high chance of developing bacteriuria. The risk of developing bacteriuria was 10 times greater with CD4 counts < 350 (86\%) compared to at CD4 counts of $>350$ cells/ $\mu \mathrm{l}$ with the $\mathrm{p}$ value of 0.01 . The mean CD4 count among the culture positives was 264 cells $/ \mu \mathrm{L}$. The observations are in accordance to the findings of Hoepelman et al $(30 \%$ with $\mathrm{P}$ value $<0.001)$ who demonstrated the greater occurrence of bacteriuria among a cohort of HIV infected patients with lower CD4 counts ${ }^{11}$. There are other studies which state that there is no significant association between HIV status, CD4 counts and UTI ${ }^{21,22}$. However an increased risk for UTI was found with increasing viral loads as reported by TA Widamer et al ${ }^{22}$.

There are numerous limitations to this study. It would have been useful to investigate the causes of all dipstick abnormalities more aggressively with better history taking and physical examination, especially in light of the unexpected levels of (sterile) leukocyturia, but this was not the aim of the initial study design. With regard to microscopic hematuria, the absence of data on the menstrual status of women is disappointing. The association between sterile pyuria and co-morbid TB, STI or other fastidious and opportunistic infections should have been explored by considering the control group of HIV-infected people without leukocyturia presenting with these conditions. Follow up of those screened with urinary abnormalities would have further strengthened the findings of this study to determine whether resolution/reversal of urinary screening abnormalities occurred with appropriate treatment (antimicrobials and ART). This applies not only to leukocyturia and microscopic hematuria but also to the persistence or resolution of proteinuria, and ASB 4 .

CONCLUSION: Individuals with HIV infection seem to be at an augmented risk of ASB, in comparison with non-HIV infected patients. Resistance to Co-trimoxazole has exceeded 35\% in uropathogens isolated from HIV patients, so this agent should no longer be used for empirical therapy. The results 
of the present study suggested that microscopic urinalysis or the nitrite dipstick alone cannot accurately predict positive urine cultures. It is recommended they can be used only as add on test and not as substitute to standard culture methods.

\section{BIBLIOGRAPHY:}

1. Baveja UK, Sokhey J. Editors. Manual on laboratory diagnosis of common opportunistic infections associated with HIV/AIDS .Government of India. National Institute of Communicable Diseases and National AIDS Control Organisation. Ministry of Health \& Family Welfare, Government of India, Nirman Bhavan, New Delhi - 110011.

2. Deokar, S.D. and M.G. Bodhankar, Studies on Emergence of Drug Resistance in HIV Associated Bacterial Urinary Tract Infections American Journal of Infectious Diseases 2009; 5 (3): 183-187.

3. MM Buzayan, Ibrahim Taher. Urinary tract infection among HIV-infected patients The Libyan Journal of Infectious Diseases 2009 July; 3(2):19 -24

4. Fabian J, Naicker S, Venter DFWillem, Baker L, Naidoo S, Paget G et al Ethnicity \& Disease, Urinary screening abnormalities in antiretroviral-naive HIV-infected outpatients and implications for management-A single-centre study in South Africa

5. Ethn Dis. 2009; 19 [Suppl 1]:S1-80-S1-85

6. MacGowan A.P, Susan Y, McCulloch, Lovering A.M. Laboratory strategy in the diagnosis of infective syndromes chapter4, Mackie \& McCartney Practical Medical Microbiology Collee J.G. Fraser A.G. Marimion B.P. Simmons A. Churchill Livingstone. Elsevier publications. Fourteenth edition: 84-90

7. SD urocolor ${ }^{\mathrm{TM}}$ Test strips for Urinalysis. Kit Insert. SD Bio Standard Diagnostics PVT.LTD Date issued: 2011-08

8. Soper, D.S. "Fisher's Exact Test Calculator for a $2 \times 2$ Contingency Table (Online Software)"2013. http://www.danielsoper.com/statcalc.

9. Staiman VR, Lowe FC. Urologic Problems in Patients with Acquired Immunodeficiency Syndrome, the Scientific World Journal (2004) 4 (S1), 427-437

10. Pead OM Ibadin, Onunu Abel, Ukoh G. Urinary tract infection in adolescent/young adults Nigerians with acquired immunedeficiency disease in Benin City. J. Med. Biomed. Res., 2006; 5(2): 55-60

11. Park J C. Buono, D, Smith D K. , Peipert J F. , Sobel J Rompalo A , et al Urinary tract infections in women with or at risk for human immunodeficiency virus infection. American Journal of Obstetrics \& Gynecology 2002 September; 187(3):581-588

12. Samuel S.O, Salami T.A.T, Adewuyi G.M, Babatope E, Ekozien M.I. Prevalence of Urinary Tract Infections among a cohort of HIV Positive Patients accessing care in a rural health centre in Nigeria J. Microbiol. Biotech. Res., 2012; 2 (4):507-510

13. De Pinho AM, Lopes GS, Ramos-Filho CF, Santos Roda R, De Oliveira MP, Halpern M, et al Urinary tract infection in men with AIDS. Genitourin Med. 1994; 70(1): 30-44.

14. Evans JK, McOwan A, Hillman RJ, Forster GE. Incidence of symptomatic urinary tract infections in HIV seropositive patients and the use of cotrimoxazole as prophylaxis against Pneumocystis carinii pneumonia Genitourin Med 1995; 71:120-122 


\section{ORIGINAL ARTICAL}

15. 14.Grabe M, Bjerklund-Johansen T.E, Botto H, Wullt B, Çek M, Naber K.G et al. Wagenlehner Guidelines on Urological Infections European Association of Urology (C) 2011

16. L.E. Nicolle. Asymptomatic bacteriuria when to screen and when to treat Infect Dis Clin N Am 2003; 7: 367-394

17. Wyatt CM, Hoover DR, Shi Q, Seaberg E, Wei C, Tien PC, et al. Microalbuminuria is associated with all-cause and AIDS mortality in women with HIV infection. J Acquir Immune Defic Syndr. 2010 Sep; 55(1):73-7

18. Gardener LI, Holmberg SD, Williamson JM, Szczech LA, Carpenter CJ Charles. Rompalo AM et al. Development of Proteinuria or elevated serum creatinine mortality in HIV infected women. Journal of Acquired Immune Deficiency Syndrome 2003 February; 32(2): 203-209

19. Inyang-Etoh P.C, Udofia G.C, Alaribe A. A. A, Udonwa N.E. Asymptomatic Bacteriuria in Patients on Antiretroviral Drug Therapy in Calabar J. Med. Sciences2009; 9 (6): 270

20. Lee L.K, Dinneen and Ahmad S. The urologist and the patient infected with human immunodeficiency virus or with acquired immunodeficiency syndrome. BJU International 2001; 88: 500-510

21. Weriebor B.C, Obi C.L, Akinyemi O, Ramalivhana N. J, Hattori T. and Okoh A. I.

22. Uropathogens isolated from HIV-infected patients from Limpopo Province, South Africa.

23. African Journal of Biotechnology 2012 June 7; 11(46): 10598-10604.

24. 21. Ojoo J, Paul F J, Batchelor M, Amid B, Kimar P J, Mwachari C, Bwayo J. Bacteriuria in a Cohort of Predominantly HIV-1 Seropositive Female Commercial Sex Workers in Nairobi, Kenya Journal of Infection 1996; 33: 33-37

25. 22. Widamer TA, Theron G, Grove D. Prevalence and risks of asymptomatic bacteriuria among HIV-positive pregnant women. Southern African Journal of Epidemiology and Infection 2010; 25(1):28 - 32 